\title{
Stereochemical Control of the Triflate Mediated Intramolecular Schmidt Reaction
}

\author{
Lars Gnägi, ${ }^{a}$ Florence Giornal, ${ }^{a}$ Harish Jangra, ${ }^{b}$ Ajoy Kapat, ${ }^{a}$ Erich Nyfeler, ${ }^{a}$ Robin Marc Schärer, ${ }^{a}$ Hen- \\ drik Zipse*b and Philippe Renaud*a
}

a) Department of Chemistry and Biochemistry, University of Bern, Freiestrasse 3

$\mathrm{CH}-3012$ Bern (Switzerland)

b) Department of Chemistry, LMU München, Butenandtstrasse 5-13

81377 München (Germany)

KEYWORDS. azide, Schmidt reaction, stereochemistry, 1,2-migration, azabicyclic compounds, alkaloids.

\begin{abstract}
The stereoselectivity of the triflate mediated intramolecular Schmidt reaction of substituted 3-(1-azidocyclohexyl)propanol derivatives leading to octahydro- $1 \mathrm{H}$-pyrrolo[1,2-a]azepine, the structural skeleton of several important families of alkaloids such as the Stemona alkaloids, has been examined. The reaction involves an initial intramolecular $\mathrm{S}_{\mathrm{N}} 2$ reaction between the azide moiety and the triflate affording an intermediate spirocyclic aminodiazonoium salt that undergoes the expected 1,2-shift/ $\mathrm{N}_{2}$-elimination followed by hydride mediated iminium salt reduction. Remarkably, chiral alcohols are converted to the azabicylic derivative with no or limited racemization. The initial asymmetric alcohol center controls the diastereoselectivity of the whole process leading to the formation of one out of the four possible diastereoisomers of disubstituted octahydro-1H-pyrrolo[1,2-a]azepine. The origin of the stereoselectivity of is rationalized based on theoretical calculations.
\end{abstract}

\section{Introduction}

In the early 1990s, Aubé ${ }^{1-5}$ and Pearson ${ }^{6}$ independently reported an intramolecular Schmidt reaction involving the reaction of alkyl azides with cationic species obtained by diverse procedures including treatment of ketones, aldehydes, hemiketals, ketals, alkenes, alcohols and epoxides with Lewis or Brønstedt acids. 7,8 This reaction was applied for the synthesis of a variety of nitrogen containing heterocycles and naturally occurring alkaloids. ${ }^{9,10}$ The regioselectivity of the carbonto-nitrogen 1,2-shift was rationalized by assuming a concerted migration mechanism over a nitrenium formation. In the concerted process, migration of a bond that is approximately antiperiplanar ${ }^{1,11}$ to the departing nitrogen in the aminodiazonium ion being preferred (Scheme $1, \mathbf{A}) .{ }^{6}$ No regioselectivity was observed for the reaction leading to indolizidine from tertiary alcohols (Scheme 1, B), this was rationalized by a non-regioselective 1,2-shift of the intermediate spirocyclic aminodiazonium salt resulting from the rearrangement of the initially formed cation followed by reaction with the azide. In such a system, the aminodiazonium salt is believed to exist as a rapidly equilibrating mixture of epimers at the nitrogen atom. ${ }^{6}$ This assumption is in accordance with calculations by Glaser, who reported that the activation barrier on the pyramidal nitrogen is low. ${ }^{7}$ The stereochemical outcome of the intramolecular Schmidt reaction has also been examined by Aubé, who discussed the preferential migration of the on-tether substituent and reported that the stereochemistry at the migrating carbon was preserved similarly to what is observed for other sextet rearrangement processes. ${ }^{8}$ More interestingly, by using chiral 1,2- and 1,3-azidoalcohols, good to excellent control of the stereochemistry could be obtained for the desymmetrization of 4-substitued cyclohexanone (Scheme 1, C). ${ }^{9,10,12}$ This process requires formation of an aminodiazonium cation bearing a chiral center next to the azido group followed by a stereoselective 1,2-shift. The $\mathrm{C}-\mathrm{C}$ bond anti to the phenyl group at the chiral center of the azidoalcohol is migrating. Assuming antiperiplanar migration suggests that the migration involves a conformation where the diazonium substituent is lying cis to the phenyl ring.

Scheme 1. Regio- and stereoselectivity of the intramolecular Schmidt reaction. 


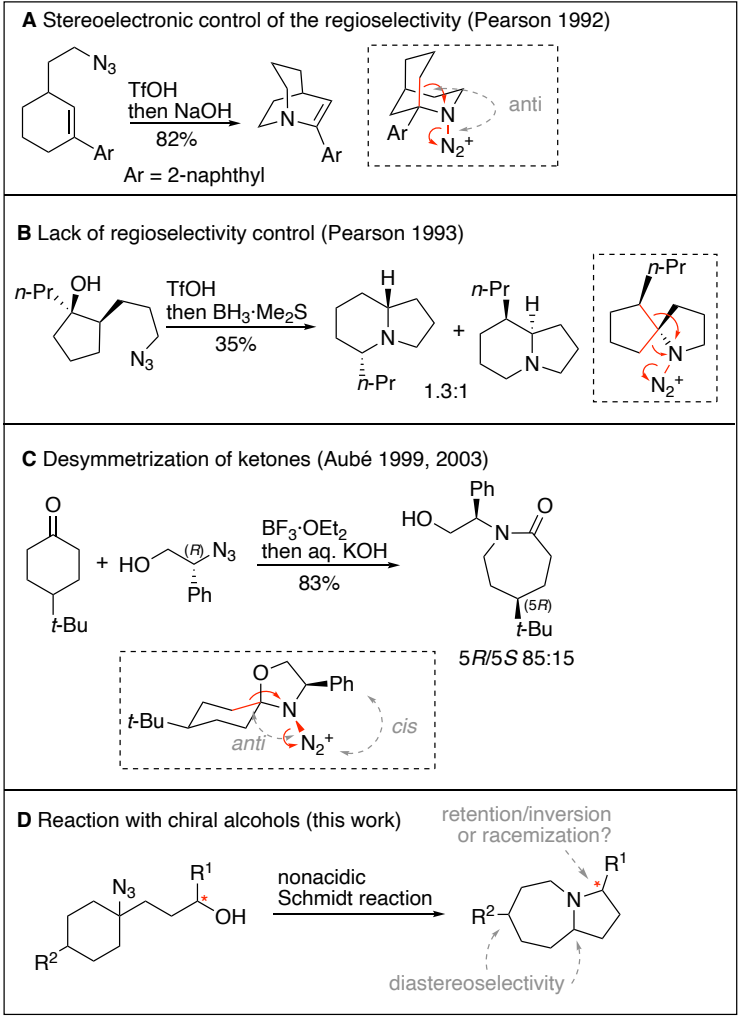

In a recent paper, we reported a modified protocol in which the Schmidt reaction is run under nonacidic conditions by converting azidoalcohols into azidotriflates that rearrange spontaneously. This approach allows to avoid rearrangement of the intermediate carbocations (see Scheme 1, B) and was used for a concise synthesis of indolizidine 167B.$^{11}$ Interestingly, racemization caused by the formation of carbocation intermediates is expected to be suppressed opening new opportunities for asymmetric Schmidt reactions involving chiral azidoalcohols. For instance, the preparation of enantiomercially enriched substituted octahydro- $1 \mathrm{H}$-pyrrolo[1,2-a]azepine, ${ }^{12}$ a skeleton found in many alkaloids natural products such as Stenoma alkaloids ${ }^{13}$ and dendrobatid frog alkaloids, ${ }^{14,15}$ is expected to be possible starting from easily available azidoalcohols such as 1-substituted 3-(1-azidocyclohexyl)propan-1-ols (Scheme 1, D). We report here that such reactions involving a chiral alcohol as unique element of asymmetry are highly diastereoselective and take place with no or very limited racemization.

\section{RESULTS AND DISCUSSION}

Reactivity of the system and 1,2-stereocontrol of the iminium reduction by an adjacent silyloxy group

The reaction conditions were optimized with the dioxolanyl acetal 1. Under our optimized conditions, ${ }^{11}$ the octahydropyrroloazepine $\mathbf{2}$ was obtained in good yield using DIBAL as a reducing agent (Scheme 2). Starting from the azidoalcohol 3 containing an asymmetric center adjacent to the azido group, a moderate stereocontrol for the hydride addition to iminium ion 4im ${ }^{+}$using $\mathrm{NaBH}_{4}$ leading to cis-4 (after desilylation) was observed. As expected, the hydride delivery is taking place anti to the bulky silyloxy group (Scheme 2, E). Increasing the control of the diastereoselectivity using DIBAL was not attempted.

Scheme 2. Formation of pyrrolo[1,2-a]azepine 2 and 4 and stereoselectivity of the reduction of the iminium salt $4 \mathrm{im}^{+}$.

Optimized reactions conditions
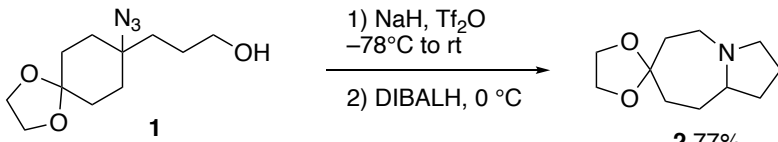

1,2-Stereocontrol of the iminium reduction<smiles>NC1(C([OH2+])CCO)CCC2(CC1)OCCO2</smiles>

3

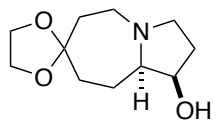

cis-4 (cis/trans 2:1)

\section{1,4-Stereocontrol of the iminium reductions and stereose- lective 1,2-shift.}

When the acetal is replaced by a phenyl group, the starting material 5 remains achiral but the iminium ion intermediate $\mathbf{6 i m}^{+}$is chiral. Therefore, the stereochemical outcome of the reduction of the iminium ion $\mathbf{6 i m}^{+}$is controlled via a 1,4-induction process. Product 6 was formed with a moderate stereocontrol when reacted with $\mathrm{NaBH}_{4}, \mathrm{NaBH}_{3} \mathrm{CN}$ and $\mathrm{LiAlH}_{4}$. An excellent cis stereocontrol was obtained with DIBAL (Table 1).

Table 1. 1,4-Stereocontrol during the Schmidt reaction converting azidoalcohol 5 to pyrrolo[1,2-a]azepine 6 .

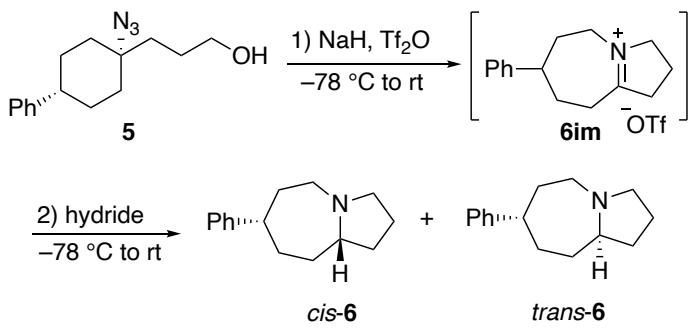

\begin{tabular}{llll}
\hline Entry & Reagent & Yield $^{\text {a }}$ & cis/trans $^{\text {b }}$ \\
\hline 1 & $\mathrm{NaBH}_{4}$ & n.d. & $67: 33$ \\
2 & $\mathrm{NaBH}_{3}(\mathrm{CN})$ & n.d & $64: 36$ \\
3 & $\mathrm{LiAlH}_{4}$ & $60 \%$ & $84: 16$ \\
4 & DIBAL & $77 \%$ & $95: 5$ \\
\hline
\end{tabular}

a) Isolated yields. ${ }^{\text {b) }}$ Determined by 1-H-NMR analysis of the crude products.

The very high cis diastereoselectivity observed for the reduction of the iminium ion $\mathbf{~ i m}^{+}(1,4$-induction) is puzzling and can potentially result from strong cation- $\pi$ interactions between 
the phenyl group and the iminium ion which effectively shields the bottom face of the iminium during the reduction favouring the anti addition of the hydride. A detailed analysis of the conformational space of cation $\mathbf{6 i m}^{+}$was performed (see supporting information for full details). All the QM results are reported at the PCM $\left(\mathrm{CH}_{2} \mathrm{Cl}_{2}\right.$,ua0)/DLPNO-CCSD(T)/cc-pVTZ//B3LYP$D 3 / 6-31 G(d)$ level of theory. The best cation- $\pi$ interaction-induced folded conformation is $12.5 \mathrm{~kJ} / \mathrm{mol}$ and $18.3 \mathrm{~kJ} / \mathrm{mol}$ higher in terms of enthalpy $\left(\Delta \mathrm{H}_{\mathrm{sol}}\right)$ and free energy $\left(\Delta \mathrm{G}_{\mathrm{sol}}\right)$ with respect to the global minimum extended conformation (Figure 1). Thus, preference for a folded conformation of iminium cation $\left(6^{-i m^{+}}\right)$due to cation- $\pi$ interactions as initially hypothesized is not supported by these results. Restricted conformational analysis of iminium salt 6im indicates that triflate anion prefers to coordinate to the concave face of the iminium intermediate. This conformation was also found to be prevalent in the solidstate structure of 6im determined by single crystal X-ray analysis (Figure 1). ${ }^{16}$ In the lowest energy conformer of iminium salt 6im, the face syn to the phenyl group is blocked by triflate anion, thus favouring the formation of the cis product through anti addition of the hydride (Model F). Calculation of transition state energies were performed to get a better understanding of the factors governing the stereochemistry of the reduction of $6 \mathrm{im}^{+}$with DIBAL. Reaction barriers calculated for the reduction of cation $6 \mathrm{im}^{+}$with DIBAL predict a $9.5 \mathrm{~kJ} \mathrm{~mol}^{-1}$ preference for the formation of cis- $\mathbf{6}$ (TS-anti) relative to trans- 6 (TS-syn) (Figure 1), which implies that the experimentally observed stereoselectivity is a kinetic phenomenon, cis-6. $\mathrm{Al}(i-\mathrm{Bu})_{2}{ }^{+}$being less stable than trans-6. $\mathrm{Al}(i-\mathrm{Bu})_{2}{ }^{+}$by $10.9 \mathrm{~kJ} \mathrm{~mol}^{-1}$ in $\mathrm{CH}_{2} \mathrm{Cl}_{2}$ (see supporting information).

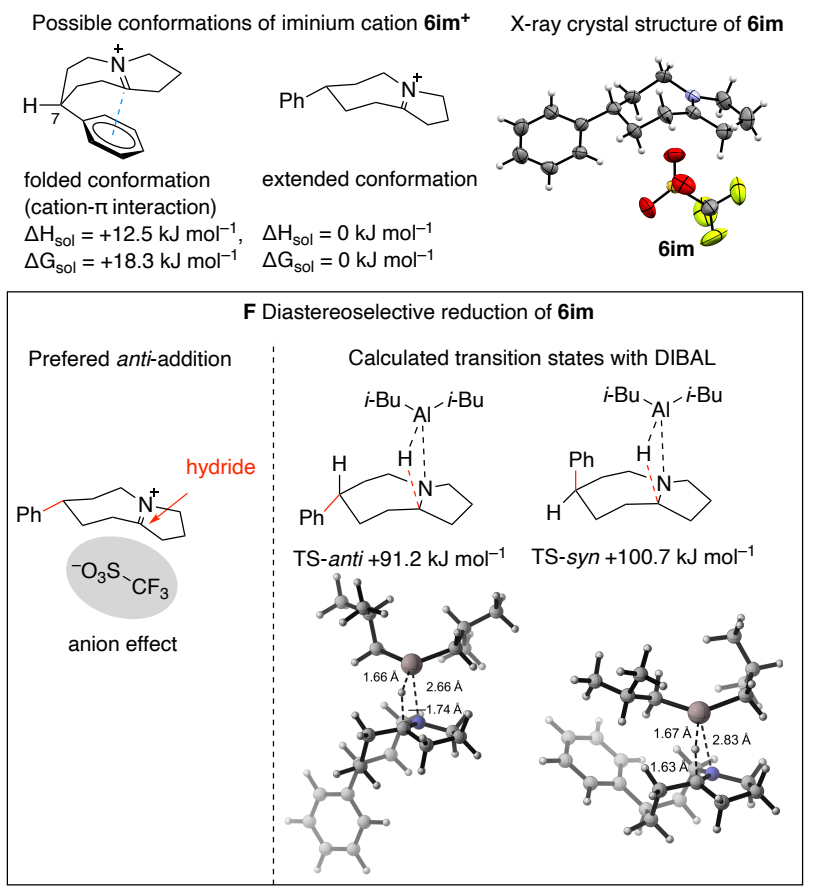

Figure 1. Conformational energetics of iminium cation 6 im $^{+}$. X-ray structure analysis of $6 \mathrm{im}$ (ellipsoids drawn at $50 \%$ probability) and proposed model for 1,4-induction during hydride addition to $6 \mathrm{im}$ as well as calculated anti and syn transition states barriers (based on best conformer) for the reaction 6im $^{+}$with DIBAL. Calculations have been performed at the $\mathrm{PCM}\left(\mathrm{CH}_{2} \mathrm{Cl}_{2}\right.$, ua0)/DLPNO-CCSD(T)/CcpVTZ//B3LYP-D3/6-31G(d) level of theory.

The Schmidt reaction with the OTBS substituted alcohol 7 containing a single asymmetric center at $\mathrm{C}(1)$ (product numbering) was investigated. The configuration of the starting material 7 was assigned by single crystal X-ray analysis of the 3,5dinitrobenzoate ester $\mathbf{7 d n b}$. $^{16}$ During the Schmidt reaction, a second chiral center at $C(7)$ is created during the 1,2-migration process and a third one at $C(9 a)$ is introduced during the final hydride addition step. Desilylation during workup eventually leads to aminoalcohol $\mathbf{8}$. When the reaction was run with DIBAL, a mixture of 2 diastereomers (out of the four possible) in a 85:15 ratio was observed. By running the final iminium reduction step with $\mathrm{NaBH}_{4}$, a single diastereomer was obtained (Scheme 3). The 3,5-dinitrobenzoate ester $\mathbf{8 d n b}$ was prepared from the major isomer of 8 by acylation with 3,5-dinitrobenzoyl chloride. Its relative $(1 R S, 7 S R, 9 a S R)$ configuration could be determined unambiguously by single crystal X-ray analysis (Scheme 3). ${ }^{16}$ Based on the relative configuration of the starting azide 7 (azido group trans to the phenyl substituent, see $X$ ray structure of $\mathbf{7 d n b}$ in Scheme 3 ) and the relative configuration of the final product $\mathbf{8}$ (see X-ray structure of $\mathbf{8 d n b}$ in Scheme 3), one can conclude that the $\mathrm{C}-\mathrm{C}$ bond anti to the OTBS group is migrating (Figure $2, \mathbf{G}$ ) and that the final hydride addition to the iminium ion $\mathbf{7 i m}^{+}$is taking place anti to the phenyl group (1,4-induction) and syn to the adjacent OTBS group.

Scheme 3. Stereochemical outcome of the intramolecular Schmidt reaction with 7. X-ray structure analysis of $7 \mathrm{dnb}$ and $8 \mathrm{dnb}$ (major diastereomer) (ellipsoids drawn at $50 \%$ probability).

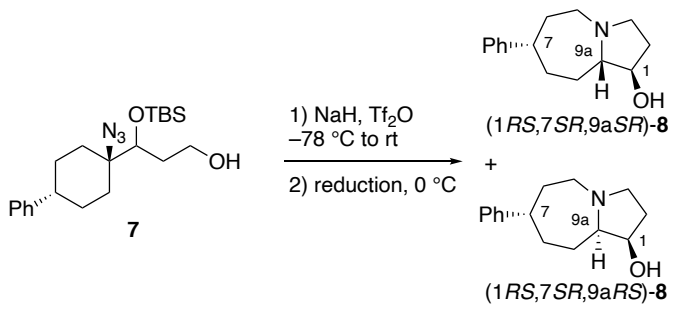

$85 \% 9 a S R / 9 a R S>98: 2 \quad\left(\mathrm{NaBH}_{4}\right)$ $68 \%$ a aSR/9aRR 85:15 (DIBALH)

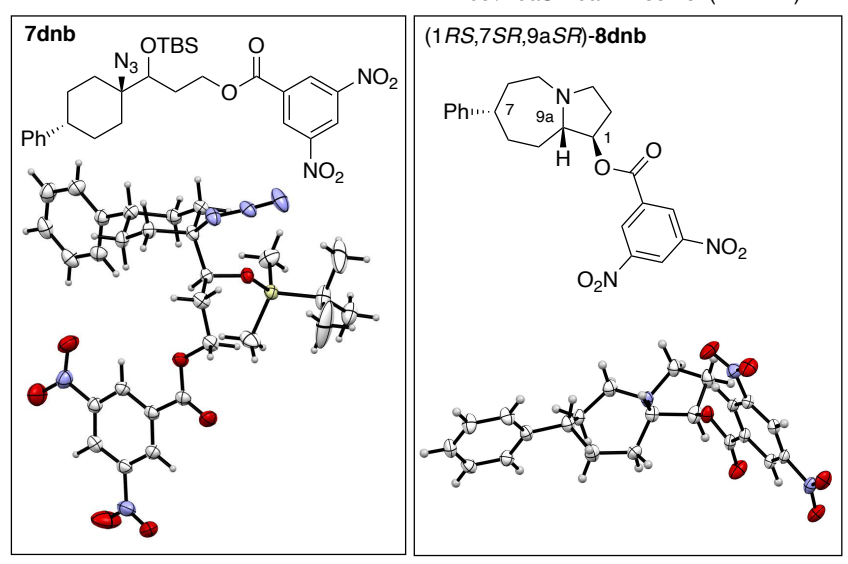

The remarkable diastereoselectivity of the 1,2-migration process is supported by reaction path calculations. Migration of the anti $\mathrm{C}-\mathrm{C}$ bond in the aminodiazonium ion $7 \mathrm{ad}^{+}$enjoys a 
barrier advantage of at least $15 \mathrm{~kJ} \mathrm{~mol}^{-1}$ (see Figure 2, G). This barrier difference is largely similar in the gas phase or in solution and may thus derive predominantly from differences in the alignment of the reacting $\mathrm{C}-\mathrm{C}$ and $\mathrm{C}-\mathrm{N}$ bonds with the surrounding substrate scaffold. It should be added that the energetic benefit of the anti over the syn transition state is completely lost upon formation of the respective iminium ions cis$8 \mathrm{im}^{+} /$trans-8im ${ }^{+}$(which are found to be isoenergetic). The final hydride addition to the iminium ion trans-8im ${ }^{+}$is taking place anti to the phenyl group (1,4-induction) and syn to the adjacent OTBS group. This process was examined through theoretical calculations in $\mathrm{CH}_{2} \mathrm{Cl}_{2}$ solution. ${ }^{17}$ The ten energetically most favourable conformations of this cation are of the extended type, which precludes control of the reduction step through a folded transition state (see supporting information). Conformational analysis of the full ion pair trans-8im, however, indicates that the silyloxy substituent present in this system directs the triflate anion exclusively to the anti (relative to OTBS or bottom) face of the iminium ion. The energetically best conformer with the triflate anion in top location is located $19.5 \mathrm{~kJ}$ $\mathrm{mol}^{-1}$ higher in energy, which is in full support of the model presented in Figure $2 \mathbf{H}$. The use of a smaller reducing agent $\left(\mathrm{NaBH}_{4}\right)$ instead of DIBAL favours the top face approach by minimizing destabilizing interactions with the OTBS group.
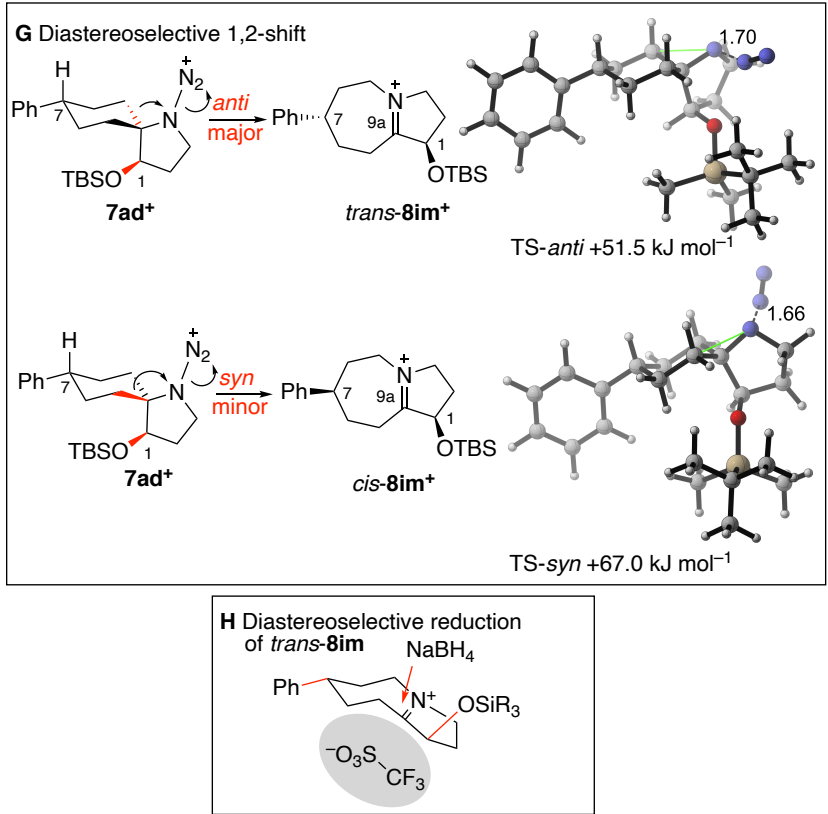

Figure 2. Rationalization of the stereochemical outcome of the conversion of $\mathbf{7}$ to $\mathbf{8}$ and transition state barriers $\left(\Delta \mathrm{G}_{\mathrm{sol}}\right.$, in $\mathrm{kJ} \mathrm{mol}^{-}$ 1) for concerted $\mathrm{C}$ to $\mathrm{N}$ bond migration (anti and syn relative to OTBS group) calculated at the $\mathrm{PCM}\left(\mathrm{CH}_{2} \mathrm{Cl}_{2}\right.$, ua0)/DLPNOCCSD(T)/cc-pVTZ//B3LYP-D3/6-31G(d) level of theory.

Stereospecificity of the Schmidt reaction involving chiral alcohols

In Pearson's version of the intramolecular Schmidt reaction, the alcohol center is converted to a cation, therefore this center cannot be used to control the absolute configuration of the final product. ${ }^{6}$ The triflate mediated version of the Schmidt reaction offers a possibility to use a chiral alcohol to control the absolute configuration of the products since the reaction most probably does not involve the formation of a cationic intermediate (at least for primary and secondary alcohols). To test this hypothesis, four different chiral alcohols 10-13 bearing a single chiral center at the alcohol position were prepared (Scheme 4). Alcohols $\mathbf{1 0}$ and $\mathbf{1 1}$ were prepared by a $\mathrm{N}, \mathrm{N}$-dibutyl-D-norephedrine ((-)-DBNE) and (+)-N-methyl ephedrine catalyzed enantioselective addition ${ }^{18-21}$ of $\mathrm{Et}_{2} \mathrm{Zn}(\mathbf{1 0})$ and phenylacetylene (11) to the aldehyde 9 prepared by oxidation of the primary alcohol 5. Brown asymmetric allylboration ${ }^{22}$ of $\mathbf{9}$ afforded the homoallylic alcohol 12. Finally, the benzylic alcohol 13 was prepared from ketone 14 via CBS-mediated enantioselective reduction. ${ }^{23}$ The absolute configurations of 10-13 were attributed based on reported stereochemical outcome of similar reactions. For compound $\mathbf{1 0}$, single crystal X-ray analysis of the 4-bromo-3-nitrobenzoate ester 10 bnb $^{16}$ confirmed its $(S)$-absolute configuration in accordance with expectations. ${ }^{24}$

Scheme 4. Preparation of chiral alcohols 10-13. X-ray structure analysis of $(S)-10 \mathrm{bnb}$ (ellipsoids drawn at $50 \%$ probability).
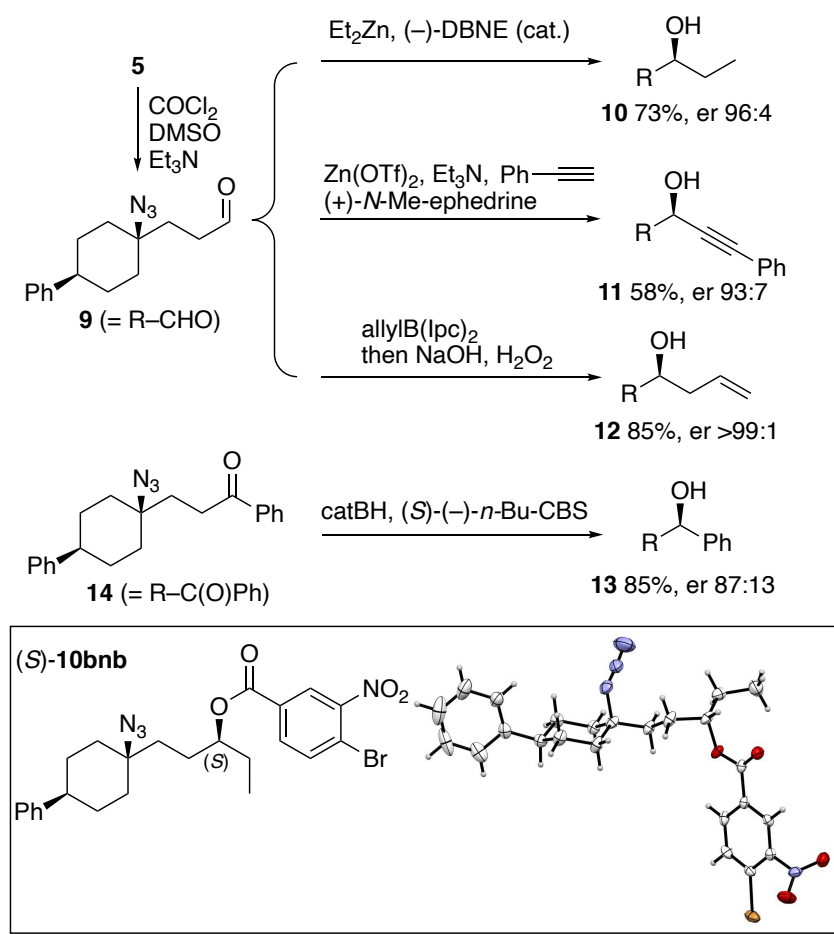

The intramolecular Schmidt reaction involving 10-13 was investigated next (Table 2). Under the standard reaction conditions, all four alcohols gave the desired bicyclic amides 15-18 in $32-85 \%$ yield as a single diastereomer. Interestingly, the enantiomeric ratio of the starting material was fully preserved for 15 and 16 and only slight loss of optical purity was observed for $\mathbf{1 7}$ and even more remarkably for $\mathbf{1 8}$ derived from the benzylic alcohol 13. The absolute configuration of $\mathbf{1 5}$ could be established using single crystal X-ray analysis of its $(R)$-mandelic acid salt (Figure 3 ). ${ }^{16}$

Table 2. Intramolecular Schmidt reaction involving enantioenriched chiral alcohols 10-13. 


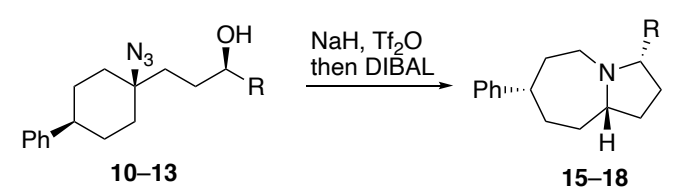

\begin{tabular}{lllllll}
\hline $\begin{array}{l}\text { En- } \\
\text { try }\end{array}$ & $\begin{array}{l}\text { Alco- } \\
\text { hol }\end{array}$ & R & er & $\begin{array}{l}\text { Prod- } \\
\text { uct }\end{array}$ & Yielda $^{\text {er }}$ & \\
\hline 1 & $\mathbf{1 0}$ & Et & $96: 4$ & $\mathbf{1 5}$ & $85 \%$ & $93: 7$ \\
2 & $\mathbf{1 1}$ & $\mathrm{PhC}=\mathrm{C}$ & $93: 7$ & $\mathbf{1 6}$ & $32 \%$ & $95: 5$ \\
3 & $\mathbf{1 2}$ & $\mathrm{CH}_{2}=\mathrm{CHCH}_{2}$ & $>99: 1$ & $\mathbf{1 7}$ & $79 \%$ & $92: 8$ \\
4 & $\mathbf{1 3}$ & $\mathrm{Ph}$ & $\mathbf{8 7 : 1 3}$ & $\mathbf{1 8}$ & $71 \%$ & $78: 22$ \\
\hline
\end{tabular}

a) Isolated yields. b) Determined by HPLC analysis using chiral

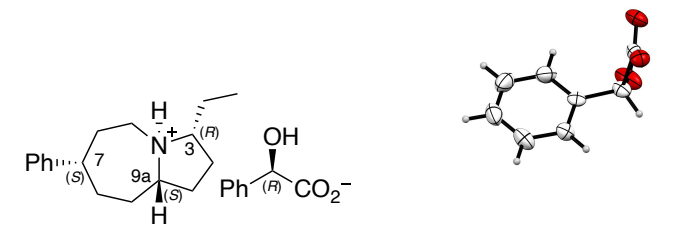

$(3 R, 7 S, 9 a S)-15 \cdot(R)-$ mandelic acid

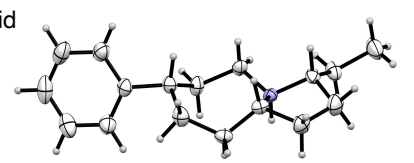

Figure 3. Determination of the absolute configuration of $(3 R, 7 S, 9 \mathrm{aS})-15$ by $\mathrm{X}$-ray structure analysis of its $(R)$-mandelic salt (ellipsoids drawn at $50 \%$ probability).

The absolute configurations $(S)$-10 and $(R)$-15 demonstrate that the intramolecular Schmidt reaction proceeds with inversion of configuration at the alcohol stereocenter. This indicates that an $\mathrm{S}_{\mathrm{N}} 2$-type mechanism is involved in the formation of the intermediate spirocyclic aminodiazonium salt 10ad+ (Figure 4, I). Even the benzylic alcohol 13 reacts mainly via an $S_{N} 2$ pathway since only limited racemization is observed. The diastereoselectivity of 1,2-shift is then controlled by the $\mathrm{C}(3)$ chiral center according to Figure $4 \mathrm{~J}$ ). Selective migration of the $\mathrm{C}-\mathrm{C}$ bond anti to the substituent at position 3 is observed. Since migration is expected to occur anti to the $\mathrm{N}-\mathrm{N}_{2}{ }^{+}$bond, it suggests that the $\mathrm{C}(3)$ substituent and the diazonium residue are cis to each other in the reactive conformation of the aminodi-

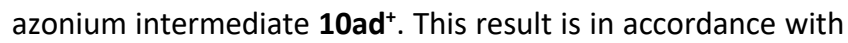
Aubé's results discussed in Scheme 1 (C), ${ }^{10}$ and also fully supported theoretical calculations of the reaction energy profiles (see supporting information). ${ }^{17}$ The anti-migration pathway 1 is found to be $8.5 \mathrm{~kJ} \mathrm{~mol}^{-1}$ more favourable than the respective syn-migration pathway (Figure 4, J). Finally, the reduction leading to the formation of the third asymmetric center at $C(9 a)$ is fully controlled by cooperative 1,4- and 1,3-induction processes from $C(3)$ and $C(7)$ (Figure 4, K).

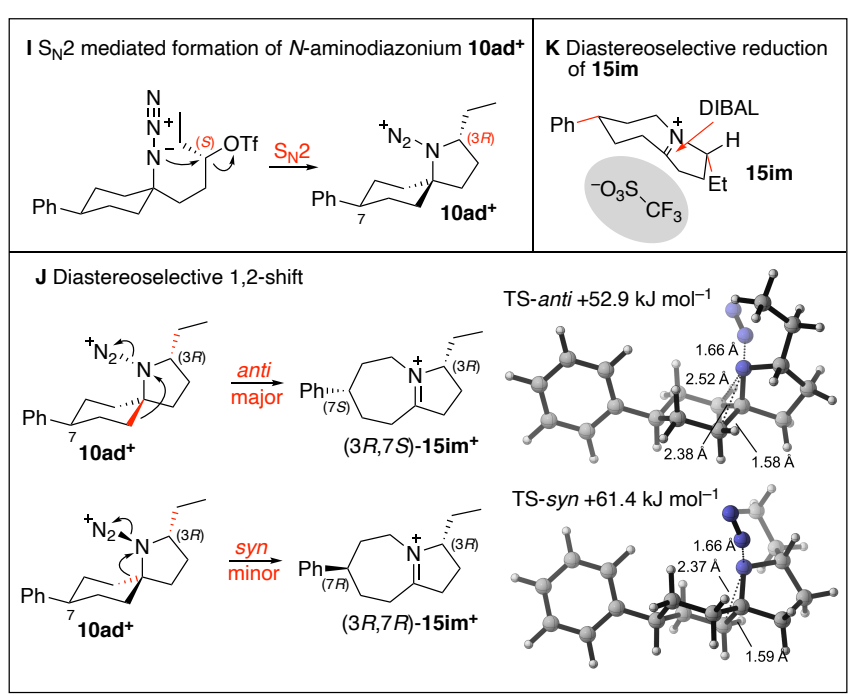

Figure 4. Stereochemical outcome of the reactions with chiral alcohol 10. Transition state barriers $\left(\Delta \mathrm{G}_{\mathrm{sol}}\right.$, in $\left.\mathrm{kJ} \mathrm{mol}^{-1}\right)$ calculated at the $\mathrm{PCM}\left(\mathrm{CH}_{2} \mathrm{Cl}_{2}\right.$, ua0)/DLPNO-CCSD(T)/cc-pVTZ//B3LYP-D3/6$31 \mathrm{G}(\mathrm{d})$ level of theory.

\section{CONCLUSION}

We have demonstrated that the triflate mediated intramolecular Schmidt reaction can be highly diastereoselective in case of symmetrical 4-substituted cyclohexyl derivatives when a chiral center is present in the carbon chain linking the azide and the alcohol. When the chirality is at the alcohol center, the process involves an initial intramolecular $S_{N} 2$ reaction between the azide moiety and the triflate. Starting from an enantiopure chiral alcohol leads to an inversion of the stereocenter with no or limited racemization depending on the substituents. Interestingly, the inverted stereocenter is then able to control the diastereoselectivity of the subsequent 1,2-alkyl shift. Since the stereochemistry of the final reduction step using a hydride source is also diastereoselective, this process allows to prepare selectively one out of the four possible diastereoisomers of disubstituted octahydro- $1 \mathrm{H}$-pyrrolo[1,2-a]azepine in a highly enantioenriched form. Application of this stereoselective process for the synthesis of naturally occurring alkaloids is currently ongoing and will be reported in due course.

\section{ASSOCIATED CONTENT}

\section{Supporting Information}

Experimental procedures and characterization of all new products, copies of NMR spectra (PDF)

Computational details (PDF)

The Supporting Information is available free of charge on the ACS Publications website.

\section{AUTHOR INFORMATION}

\section{Corresponding Authors}

Hendrik Zipse - Department of Chemistry, LMU München, Butenandtstrasse 5-13, 81377 München (Germany)

Philippe Renaud - Department of Chemistry and Biochemistry, University of Bern, Freiestrasse 3, CH-3012 Bern (Switzerland) 
https://orcid.org/0000-0002-9069-7109

\section{Authors}

Lars Gnägi - Department of Chemistry and Biochemistry, University of Bern, Freiestrasse 3, CH-3012 Bern (Switzerland) https://orcid.org/0000-0003-4610-7252

Florence Giornal - Department of Chemistry and Biochemistry, University of Bern, Freiestrasse 3, CH-3012 Bern (Switzerland)

Harish Jangra - Department of Chemistry, LMU München, Butenandtstrasse 5-13, 81377 München (Germany)

Ajoy Kapat - Department of Chemistry and Biochemistry, University of Bern, Freiestrasse 3, $\mathrm{CH}-3012$ Bern (Switzerland)

https://orcid.org/0000-0002-3236-4349

Erich Nyfeler - Department of Chemistry and Biochemistry, University of Bern, Freiestrasse 3, CH-3012 Bern (Switzerland)

Robin. M. Schärer - Department of Chemistry and Biochemistry, University of Bern, Freiestrasse 3, CH-3012 Bern (Switzerland)

\section{Funding Sources}

The Swiss National Science Foundation (Project 200020_172621) is gratefully acknowledged for financial support.

\section{Notes}

Any additional relevant notes should be placed here.

\section{ACKNOWLEDGMENT}

Konrad Uhlmann is acknowledged for performing GC and HPLC analyses. We thank the group of Chemical Crystallography of the University of Bern (Prof. Dr. P. Macchi) for the X-ray structure solution as well as PD Dr. Simon Grabowsky, Dr. Michal Andrzejewski and Dr. Michelle Ernst for their help in solving X-ray crystal structures. We would also like to thank the Leibniz Supercomputing Centre (www.Irz.de) for generous allocation of computational resources.

\section{ABBREVIATIONS}

TBS, (tert-butyl)dimethylsilyl; DIBAL, diisobutylaluminum hydride.

\section{REFERENCES}

(1) Aubé, J.; Milligan, G. L. Intramolecular Schmidt Reaction of Alkyl Azides. J. Am. Chem. Soc. 1991, 113 (23), 8965-8966. https://doi.org/10.1021/ja00023a065.

(2) Pearson, W. H.; Schkeryantz, J. M. An Intramolecular, Schmidt Reaction of an Alkyl Azide with a Carbocation. The Generation and Rearrangement of a Conformationally Restricted Secondary Aminodiazonium Ion. Tetrahedron Lett. 1992, 33 (37), 5291-5294. https://doi.org/10.1016/S00404039(00)79075-7.

(3) Grecian, S.; Aubé, J. Schmidt Rearrangement Reactions with Alkyl Azides. In Organic Azides; John Wiley \& Sons, Ltd, 2010; pp 191-237. https://doi.org/10.1002/9780470682517.ch7.

(4) Nyfeler, E.; Renaud, P. Intramolecular Schmidt Reaction: Applications in Natural Product Synthesis. CHIMIA 2006, 60, 276-284. https://doi.org/10.2533/000942906777674714.

(5) Hoffman, R. V.; Kumar, A. Cationic Carbon-to-Nitrogen Rearrangements in N-(Arylsulfonoxy)Amines. J. Org. Chem. 1985, 50 (11), 1859-1863. https://doi.org/10.1021/jo00211a014.

(6) Pearson, W. H.; Walavalkar, R.; Schkeryantz, J. M.; Fang, W. K.; Blickensdorf, J. D. Intramolecular Schmidt Reactions of Azides with Carbocations: Synthesis of Bridged-Bicyclic and Fused-Bicyclic Tertiary Amines. J. Am. Chem. Soc. 1993, 115 (22), 10183-10194. https://doi.org/10.1021/ja00075a038.

(7) Glaser, R.; Choy, G. S. C. Heterosubstituted Diazonium lons $\mathrm{FNN}+$, $\mathrm{HONN+}$, and $\mathrm{H} 2 \mathrm{NNN+}$. Automerization,

Dediazoniation, and Deprotonation. J. Phys. Chem. 1991, 95 (20), 7682-7693. https://doi.org/10.1021/j100173a025.

(19) Soai, K.; Kawase, Y.; Oshio, A. Enantioselective Phenylation of Prochiral Aldehydes Using a Kinetically Formed Chiral Complex between Grignard-Zinc Halide Reagent and N,N-Dibutylnorephedrine. J. Chem. Soc. Perkin 1 1991, No. 6, 16131615. https://doi.org/10.1039/P19910001613. 
(20) Soai, K.; Niwa, S. Enantioselective Addition of Organozinc Reagents to Aldehydes. Chem. Rev. 1992, 92 (5), 833-856. https://doi.org/10.1021/cr00013a004.

(21) Soai, K.; Hayase, T.; Takai, K.; Sugiyama, T. Asymmetric Synthesis of Chiral Secondary Alcohols with Isopropyl Substituents by the Catalytic Enantioselective Addition of Diisopropylzinc to Aldehydes Using N,N-Dialkylnorephedrines as Chiral Catalysts. J. Org. Chem. 1994, 59 (25), 7908-7909. https://doi.org/10.1021/jo00104a057.

(22) Brown, H. C.; Jadhav, P. K. Asymmetric Carbon-Carbon Bond Formation via B-Allyldiisopinocampheylborane. Simple Synthesis of Secondary Homoallylic Alcohols with Excellent
Enantiomeric Purities. J. Am. Chem. Soc. 1983, 105 (7), 20922093. https://doi.org/10.1021/ja00345a085.

(23) Corey, E. J.; Helal, C. J. Reduction of Carbonyl Compounds with Chiral Oxazaborolidine Catalysts: A New Paradigm for Enantioselective Catalysis and a Powerful New Synthetic Method. Angew. Chem. Int. Ed. 1998, 37 (15), 1986-2012. https://doi.org/10.1002/(SICl)1521-

3773(19980817)37:15<1986::AID-ANIE1986>3.0.CO;2-Z.

(24) Soai, K.; Shibata, T. Design of Chiral Catalysis and Asymmetric Autocatalysis for the Enantioselective Additions of Organozinc Reagents. J. Synth. Org. Chem. Jpn. 1997, 55 (11), 994-1005. https://doi.org/10.5059/yukigoseikyokaishi.55.994. 